\title{
Les mémoires des futurs agrégés : un observatoire et un levier des savoirs
}

The Thesis of the Future Qualified Teachers: An Observatory and a Lever of the Knowledge

\section{Caroline Scheepers}

\section{(2) OpenEdition}

\section{Journals}

\section{Édition électronique}

URL : http://journals.openedition.org/pratiques/2111

DOI : 10.4000/pratiques. 2111

ISSN : 2425-2042

\section{Éditeur}

Centre de recherche sur les médiations (CREM)

Édition imprimée

Date de publication : 5 juin 2014

\section{Référence électronique}

Caroline Scheepers, "Les mémoires des futurs agrégés : un observatoire et un levier des savoirs », Pratiques [En ligne], 161-162 | 2014, mis en ligne le 05 juin 2014, consulté le 19 avril 2019. URL : http:// journals.openedition.org/pratiques/2111 ; DOI : 10.4000/pratiques.2111

Ce document a été généré automatiquement le 19 avril 2019

(c) Tous droits réservés 


\title{
Les mémoires des futurs agrégés : un observatoire et un levier des savoirs
}

The Thesis of the Future Qualified Teachers: An Observatory and a Lever of the Knowledge

\author{
Caroline Scheepers
}

\author{
«Parler avec les mots des autres, \\ ça doit être ça la vraie liberté » \\ La maman et la putain, Jean Eustache, 1973.
}

\section{Introduction}

Quels sont les modèles didactiques dominants dans les discours des formateurs d'enseignants? Peut-on mettre au jour les discours tenus par ces formateurs à propos de l'écriture? Quelle place l'écriture tient-elle dans les plans de formation? Autant de questions formulées par les responsables de ce numéro et que la présente contribution se propose d'envisager. Ces interrogations sont, de mon point de vue, décisives, car elles éclairent notamment la délicate problématique de la transposition des savoirs scientifiques en savoirs enseignables et enseignés. À la suite de R. Hofstetter et de B. Schneuwly (2009: 16), je considère que les savoirs s'inscrivent au centre des instituts de formation et, par conséquent, du mandat des professionnels qui y interviennent : les savoirs différencient la formation du compagnonnage, axé sur le "mimétisme dans le faire en situation » et visant l'élaboration de savoirs professionnels généralisables. C'est dire l'enjeu de la problématique qui sous-tend cet article. Les chercheurs genevois (Hofstetter \& Schneuwly, 2009: 17-18) distinguent les savoirs à enseigner (ceux qui font l'objet du travail des formateurs) et les savoirs pour enseigner (ceux qui constituent les outils de leur travail). 
2 Identifier les savoirs qui circulent dans les instituts de formation à propos de l'écriture peut donner lieu à des options méthodologiques diverses : conduire des entretiens avec les formateurs, les maitres de stage, les étudiants ou les enseignants tout juste diplômés, décrire et analyser les curriculums de formation (Caprace, 2011), étudier les notes de cours, filmer des séances de cours... L'orientation prise ici est la suivante : analyser en Fédération Wallonie-Bruxelles les travaux de fin d'études (TFE) élaborés par les étudiants au terme de leur cursus académique sous la supervision d'un formateur dans le but d'y déceler les savoirs construits et mobilisés par les "presque enseignants ». Équivalents belges des mémoires professionnels français, les TFE constituent par définition «des écrits de savoirs ", car ils s'apparentent à la fois à des écrits réflexifs et à des écrits scientifiques en formation. Dès lors, les savoirs y occupent une place de choix. Pour ce faire, a été constitué un corpus de 47 TFE, recueillis dans trois hautes écoles distinctes, volumineux corpus interrogé par le prisme des savoirs qui, dans une perspective vygtoskienne, s'y construisent et s'y révèlent dans le même temps (Vygotski, 1934-1997).

\section{Les travaux de fin d'études, un pacte scriptural complexe}

3 Le genre TFE repose sur un pacte scriptural complexe qu'il convient d'élucider quelque peu. Il est proche des mémoires professionnels français à propos desquels ont été menées de très nombreuses études, recensées notamment par J. Crinon et M. Guigue (2006), enrichissant ainsi considérablement le champ des littéracies académiques.

4 Comme son nom l'indique, le TFE conclut un cursus de formation, en l'occurrence, académique: il a donc une valeur certificative, conclusive, il constitue la dernière épreuve du parcours en haute école. Il représente une forme de rite de passage. Dans le TFE, l'étudiant est invité à mener ce qui s'apparente, toutes proportions gardées, à une recherche scientifique: il problématise au départ d'un sujet qui lui tient à cœur, il conceptualise son propos en élaborant un cadre théorique, il mène généralement une expérimentation didactique dans sa classe de stage, expérimentation fondée sur la littérature publiée, laquelle est supposée étayer l'interprétation des incidences de son intervention. Potentiellement en tout cas, les TFE constituent dès lors un levier et un observatoire privilégiés des savoirs mobilisés et façonnés par l'étudiant, mais aussi, indirectement, des savoirs qui ont circulé dans la haute école. Car dans le TFE, le scripteur est tenu de secondariser sa pratique professionnelle (Bautier \& Goigoux, 2004), les savoirs étayant ce processus de distanciation.

Formellement créées en 1995, les hautes écoles sont des établissements offrant un enseignement supérieur de type court (trois ans) ou long (cinq ans) à caractère professionnalisant. La principale mission qui incombe aux hautes écoles est d'assurer la formation initiale dans différents secteurs, elles peuvent accessoirement organiser la recherche appliquée et la formation continuée. Les formateurs qui y enseignent sont dans leur très grande majorité titulaires d'une licence ou d'un master universitaire (équivalent à un $\mathrm{Bac}+4$ ou 5 français) et d'un titre pédagogique (l'agrégation et/ou le CAPAES - Certificat d'aptitude pédagogique approprié à l'Enseignement supérieur). Très rares sont les formateurs titulaires d'un doctorat, plus rares encore sont ceux dont le doctorat est valorisé, sur le plan salarial notamment. Bien peu de formateurs en haute école effectuent des recherches scientifiques. Les catégories pédagogiques des hautes écoles 
assurent en trois ans la formation initiale des futurs instituteurs du maternel, du primaire et du secondaire inférieur (le collège français) ${ }^{1}$. L'université prend en charge en cinq ans la formation initiale des enseignants officiant dans les trois dernières années du secondaire (le lycée français). Le modèle y est consécutif : à une formation scientifique à caractère disciplinaire succède une formation pédagogique, laquelle comporte un volume horaire de 300 heures. Dans les hautes écoles, le modèle est simultané : sont enseignées de concert compétences disciplinaires, didactiques ou pédagogiques.

Dès lors, la plupart des formateurs n'ont jamais enseigné en maternelle, en primaire ou au collège, mais, à de rares exceptions près, ils n'ont pas davantage de doctorat ou ne reçoivent pas de formation véritablement spécifique à la formation d'enseignants. Ces formateurs sont supposés assurer 480 heures annuelles de cours, de supervision de stages ou de TFE. Aucun programme officiel ne définit le contenu des cours à dispenser et aucune inspection n'intervient dans les établissements.

7 Dans cette contribution, nous nous pencherons plus particulièrement sur les TFE produits par les futurs enseignants de collèges, appelés les AESI (agrégés de l'enseignement secondaire inférieur). Une particularité de ce public est qu'une grande majorité d'entre eux se sont essayés en vain préalablement à des études universitaires : l'inscription en haute école relève donc souvent d'un second choix. Beaucoup aspiraient à enseigner au secondaire supérieur: le salaire y est plus important, la fonction parait plus "prestigieuse »... Nous étudierons particulièrement les TFE des futurs enseignants de français consacrés à l'écriture : on peut supposer que beaucoup d'entre eux ont d'abord entamé des études universitaires en langues et lettres françaises et romanes. Pourquoi étudier les TFE de ce public spécifique? Ce choix m'a paru stratégique pour plusieurs raisons. D'une part, j'ai déjà conduit de nombreuses études à propos des TFE des futurs instituteurs ou des mémoires des futurs professeurs du secondaire supérieur (voir, entre autres, Scheepers, 2009, 2013). D'autre part, les étudiants sont formés à l'université par des professeurs qui sont par ailleurs des chercheurs: on peut donc raisonnablement penser qu'il y a équivalence entre les savoirs curriculaires, donc enseignés, et les savoirs scientifiques, donc construits dans la noosphère de chercheurs. Ce n'est pas le cas des futurs enseignants de collèges : il est alors possible qu'émergent des constats différents. En outre, comme l'écrit H.-E. Tenorth (2009: 105), les enseignants du primaire ont une culture spécifique par rapport aux enseignants du secondaire. Les premiers mettent en avant des savoirs professionnels de nature didactique ou pédagogique qui contribuent à définir leur culture, tandis que les seconds sont davantage orientés vers les savoirs académiques, scientifiques, leur cursus se revendiquant d'abord de spécialisations disciplinaires, au détriment des savoirs didactiques ou pédagogiques. Qu'en est-il des savoirs construits dans leur TFE par les futurs AESI, supposés soucieux des savoirs savants, mais inscrits, parfois bon gré mal gré, dans une formation professionnalisante assurée par des formateurs qui ne peuvent pas vraiment se prévaloir d'une expertise scientifique ou d'une expérience d'enseignement en collège? Toutes ces précisions contextuelles sont indispensables pour appréhender de façon pertinente les données qui seront étudiées ici et pour discuter les résultats qu'elles suggèrent.

Comment caractériser les savoirs forgés et mobilisés par les étudiants dans leur TFE ? Il me semble opératoire de recourir à une typologie, certes classique, mais fonctionnelle: pourraient donc être distingués les savoirs scientifiques (produits par des chercheurs), les savoirs curriculaires (diffusés par les formateurs), les savoirs décrétaux (énoncés dans les textes législatifs), les savoirs expérienciels (formulés au départ du travail en classe). Au 
sein des savoirs scientifiques coexistent les savoirs disciplinaires (pour le dire vite, les savoirs à enseigner, les objets d'enseignement), didactiques (les savoirs relatifs à l'enseignement d'une discipline) et pédagogiques (les savoirs plus transversaux liés à l'enseignement). Bien entendu, cette typologie est imparfaite, le partage entre les savoirs n'est guère aussi facile qu'il n'y parait. Des redondances, des zones de recouvrement existent. Par ailleurs, une même personne peut selon les cas écrire un article scientifique, collaborer à la conception d'un programme d'enseignement, assumer un cours... Cependant, la typologie adoptée s'est avérée suffisante pour notre propos. Par conséquent, quels savoirs scientifiques, curriculaires, décrétaux et expérienciels soustendent les TFE consacrés à l'écriture des futurs AESI? La recherche s'appuie sur plusieurs hypothèses. Est postulé tout d'abord un décalage entre le foisonnement extrême des recherches didactiques en lien avec l'écriture et les savoirs dont font état les TFE, sans doute focalisés autour de quelques ouvrages emblématiques: Y. Reuter (2000), J. Jolibert (2007)... Je suppose que les savoirs curriculaires sont en revanche bien présents au cœur des TFE : ils pourraient s'apparenter aux doxas didactiques qui circulent dans les hautes écoles. Quant aux savoirs décrétaux, il me semble qu'ils sont régulièrement exhibés par les scripteurs pour valider leurs choix didactiques. Enfin, j'avance l'hypothèse selon laquelle les savoirs expérienciels sont nombreux, fins et précis, résultant d'un processus réflexif, mais peu articulés aux savoirs scientifiques.

9 Mais le simple inventaire des savoirs que recèlent les TFE ne suffit guère. Il convient d'articuler l'identification fine des savoirs à la problématique de la polyphonie discursive, le TFE étant un genre éminemment dialogal. Ainsi, quelle est la proportion des discours d'autrui et leur degré de fonctionnalité (Boch \& Grossmann, 2001) ? Le recours au discours d'autrui fonctionne-t-il par accumulation ou restriction? Sont-ils simplement exhibés ou sous-tendent-ils réellement le processus de recherche? Quel est le degré d'insertion des énoncés produits par autrui: sont-ils bien intégrés aux propos de l'énonciateur ou s'y juxtaposent-ils sans plus? Quel est le degré d'attribution des discours d'autrui : autrement dit, l'étudiant recourt-il au plagiat ou indique-t-il précisément les emprunts effectués? Comment caractériser le mode de référencialisation privilégié des étudiants ? F. Boch et F. Grossmann (2002 : 44-45) distinguent à ce propos l'évocation de la reformulation et de la citation. Dans quelle mesure les discours intertextuels (la littérature publiée) interagit-elle avec les discours infratextuels (les données empiriques) (Guibert, 2001) ? A-t-on affaire à une polyphonie plutôt polémique ou consensuelle (De Nuchèze, 1998) ou, en d'autres mots, quel est le degré d'adhésion du scripteur aux discours mentionnés?

Sur un plan méthodologique, j'ai contacté six directeurs de Catégories pédagogiques en haute école afin d'obtenir leur autorisation pour accéder aux TFE. Trois ont répondu positivement ${ }^{2}$, trois autres n'ont pas donné suite. Les trois établissements, qui resteront anonymes, relèvent de réseaux d'enseignement différents et sont contrastés sur le plan géographique, sur le plan du recrutement estudiantin également, même si l'étude ne peut être qualifiée d'exhaustive ou de représentative. Je me suis rendue en bibliothèque, laquelle ne conserve dans certains établissements que les TFE crédités d'une note équivalente ou supérieure à quatorze. Il faut insister sur le fait que les bibliothèques visitées sont bien fournies, comportant des ouvrages pertinents, récents et diversifiés, permettant l'accès à des revues didactiques ou pédagogiques de qualité (Pratiques, Les Cahiers pédagogiques...). Certaines hautes écoles se situent juste à côté de bibliothèques universitaires ou publiques richement dotées, ce qui facilite encore la recherche 
documentaire dont doivent s'acquitter les étudiants... Je me suis mise en quête de TFE consacrés à l'écriture. Les titres sont parfois significatifs, car ils intègrent le mot «écriture » parfois non: en effet, il s'avère que l'écriture peut s'avérer centrale dans un TFE axé sur le concept de temporalité ou sur la littérature enfantine. Mes recherches montrent par ailleurs qu'on peut noter un relatif équilibre entre les TFE consacrés à la lecture, à l'écriture ou à l'oralité. A dès lors été constitué un corpus de 47 TFE, les plus anciens ayant été déposés en 2002 et les plus récents en juin 2013, ce qui confère à l'étude un caractère relativement diachronique. Le tableau qui suit donne à voir les titres des TFE qui ont été analysés. Ici aussi, les données restent anonymes, pour des raisons déontologiques évidentes.

Tableau 1. Les titres des TFE analysés.

\begin{tabular}{|c|c|}
\hline Années & Titre \\
\hline \multirow{3}{*}{ 2001-2002 } & Au croisement de la métacognition et de l'écriture \\
\hline & $\begin{array}{l}\text { Comment passer du modèle transmissif au modèle constructiviste? ou Comment rendre } \\
\text { l'élève du Rwanda autonome dans ses apprentissages? }\end{array}$ \\
\hline & $\begin{array}{l}\text { Comment et pour quoi amener les élèves de l'enseignement secondaire liégeois à prendre } \\
\text { conscience de leurs racines par le biais de la création littéraire? }\end{array}$ \\
\hline \multirow{7}{*}{$2002-2003$} & Le récit de voyage: un outil pour la reconnaissance de l'altérité \\
\hline & $\begin{array}{l}\text { Comment faire modifier le rapport des jeunes aux stéréotypes de l'amour à travers un } \\
\text { ensemble textuel? }\end{array}$ \\
\hline & $\begin{array}{l}\text { La structuration chronologique au service de la communication. Comment aider les élèves à } \\
\text { percevoir l'organisation temporelle dans la langue écrite et orale afin de leur permettre une } \\
\text { construction nuancée de la notion de chronologique au sens le plus large du terme? }\end{array}$ \\
\hline & $\begin{array}{l}\text { Comment rendre un élève acteur, actif et autonome dans le cadre d'un apprentissage en } \\
\text { français? }\end{array}$ \\
\hline & $\begin{array}{l}\text { La littérature de jeunesse au service de la Mémoire. Comment et pourquoi aborder la Shoah } \\
\text { avec les élèves à travers la littérature de jeunesse? }\end{array}$ \\
\hline & L'élaboration d'une revue par des adolescents pour des adolescents : quels bénéfices? \\
\hline & L'expression des émotions à travers les ateliers d'écriture \\
\hline \multirow{2}{*}{$2003-2004$} & Comment et pourquoi expliquer l'intertextualité à travers la publicité? \\
\hline & $\begin{array}{l}\text { Comment redonner le gout de lire des romans à des adolescents en se basant sur les contextes } \\
\text { social et physique? }\end{array}$ \\
\hline \multirow{2}{*}{ 2004-2005 } & $\begin{array}{l}\text { Tout l'écrit, les SMS... Le langage SMS est-il un outil efficace pour libérer l'imagination des } \\
\text { élèves dans le cadre d'un atelier d'écriture? }\end{array}$ \\
\hline & $\begin{array}{l}\text { La prise en charge de façon autonome des élèves dans le domaine de l'orthographe, afin } \\
\text { d'améliorer celle-ci, peut-elle être efficace? }\end{array}$ \\
\hline
\end{tabular}




\begin{tabular}{|c|c|}
\hline \multirow{6}{*}{$2005-2006$} & $\begin{array}{l}\text { Un journal scolaire au service de la revalorisation d'élèves de deuxième année de } \\
\text { l'enseignement professionnel? }\end{array}$ \\
\hline & $\begin{array}{l}\text { Comment permettre aux élèves d'améliorer leur rédaction d'une critique de peintures } \\
\text { surréalistes dans le cadre d'un projet? }\end{array}$ \\
\hline & Développer un appétit d'écriture sur soi à travers la création de «blogs" personnels? \\
\hline & $\begin{array}{l}\text { L'enseignement-apprentissage du vocabulaire en français langue maternelle au deuxième } \\
\text { degré de l'enseignement secondaire }\end{array}$ \\
\hline & Comment aborder la presse people dans l'enseignement qualifiant? \\
\hline & $\begin{array}{l}\text { Le haiku en classe de français. Déconstruire les stéréotypes de la poésie occidentale pour } \\
\text { s'initier à l'art de l'éphémère }\end{array}$ \\
\hline \multirow{6}{*}{$2006-2007$} & $\begin{array}{l}\text { Comment intégrer le langage SMS dans un cours de français du premier degré comme } \\
\text { déclencheur d'apprentissage? }\end{array}$ \\
\hline & Du SMS au conte... \\
\hline & Le théâtre au service des compétences Lire et Écrire \\
\hline & $\begin{array}{l}\text { Le plaisir des contes... Quelles démarches permettent d'améliorer le rapport des jeunes aux } \\
\text { contes? }\end{array}$ \\
\hline & $\begin{array}{l}\text { Comment développer un appétit d'écriture de type poétique et de genre autobiographique } \\
\text { grâce au slam? }\end{array}$ \\
\hline & $\begin{array}{l}\text { Écrire un poème «à la manière de Francis Ponge " peut-il aider les élèves à améliorer leurs } \\
\text { compétences lexicales? }\end{array}$ \\
\hline \multirow[b]{2}{*}{$2007-2008$} & Le manga en classe de français. Vers une approche différente de la bande dessinée \\
\hline & $\begin{array}{l}\text { Comment l'orthographe s'enseigne-t-elle en première année de l'enseignement secondaire } \\
\text { général ? Différencie-t-on l'apprentissage pour les enfants dysorthographiques? }\end{array}$ \\
\hline 2008-2009 & $\begin{array}{l}\text { Équation conjuguée, verbe multiplié... ou quels sont les liens entre les mathématiques et la } \\
\text { maitrise de la langue française dans l'enseignement }\end{array}$ \\
\hline \multirow{4}{*}{$2009-2010$} & $\begin{array}{l}\text { Les intelligences multiples au cœur de la différenciation de l'apprentissage. Comment } \\
\text { améliorer les résultats en lecture et en écriture des élèves en utilisant les intelligences } \\
\text { multiples? }\end{array}$ \\
\hline & $\begin{array}{l}\text { Quelles compétences particulières le recours au traitement de texte permet-il de développer } \\
\text { dans le cadre de l'écriture d'une énigme criminelle? }\end{array}$ \\
\hline & $\begin{array}{l}\text { Construire des apprentissages en lecture, écriture et oralité à partir de l'adaptation } \\
\text { cinématographique de textes littéraires. Comment développer des compétences scripturales, } \\
\text { orales, de lecture en travaillant l'adaptation cinématographique de textes littéraires dans le } \\
\text { cadre du cours de Français }\end{array}$ \\
\hline & Comment susciter de manière ludique l'envie d'écrire chez les adolescents hospitalisés? \\
\hline
\end{tabular}




\begin{tabular}{|c|c|}
\hline & Le journal intime peut-il aider l'élève à construire son identité? \\
\hline \multirow{2}{*}{$2010-2011$} & $\begin{array}{l}\text { En quoi les textes littéraires peuvent-ils permettre aux élèves de s'exprimer de façon critique } \\
\text { sur la vie sentimentale de leur époque? }\end{array}$ \\
\hline & $\begin{array}{l}\text { Comment aborder la littérature épistolaire amoureuse en quatrième technique de } \\
\text { qualification? }\end{array}$ \\
\hline \multirow{7}{*}{ 2011-2012 } & $\begin{array}{l}\text { Quel(s) moyen(s) pédagogique(s) mettre en place pour faire évoluer les représentations } \\
\text { mentales du théâtre chez les élèves du } 2^{e} \text { degré professionnel? }\end{array}$ \\
\hline & $\begin{array}{l}\text { En quoi la rédaction d'un journal intime et l'écriture de soi peut-elle aider les élèves à se } \\
\text { sentir mieux? }\end{array}$ \\
\hline & $\begin{array}{l}\text { Achève-moi, lecteur! Comment utiliser les fins ouvertes en littérature de jeunesse pour } \\
\text { enseigner la lecture et la promouvoir? }\end{array}$ \\
\hline & Comment enseigner le vocabulaire de manière efficace? Propositions didactiques \\
\hline & Comment développer la créativité des élèves lors d'ateliers d'écriture? \\
\hline & Quand les journalistes nous apprennent à enquêter... \\
\hline & $\begin{array}{l}\text { La maitrise de la langue écrite. L'influence du profil socio-économique de l'élève sur sa } \\
\text { maitrise de la langue écrite }\end{array}$ \\
\hline \multirow{4}{*}{$2012-2013$} & $\begin{array}{l}\text { L'outil portfolio: un moyen efficace pour améliorer l'expression orale grâce aux jeux de } \\
\text { rôles? }\end{array}$ \\
\hline & $\begin{array}{l}\text { Comment aider les élèves à conjuguer correctement les verbes et à choisir de manière } \\
\text { adéquate les temps verbaux dans leurs productions? }\end{array}$ \\
\hline & $\begin{array}{l}\text { Quels sont les effets possibles de l'utilisation des TIC dans l'enseignement d'une cuvre de } \\
\text { littérature classique? }\end{array}$ \\
\hline & $\begin{array}{l}\text { Le journal des apprentissages. À quelles conditions le journal des apprentissages permet-il } \\
\text { aux élèves de progresser? }\end{array}$ \\
\hline
\end{tabular}

11 Concrètement, l'analyse a tout d'abord consisté à passer au crible la bibliographie ${ }^{3}$ afin d'inventorier et de classer les références listées. Dans un deuxième temps, la lecture du cadre théorique a complété les premières observations, tandis que les savoirs dits expérienciels étaient plutôt repérables dans les analyses réflexives qui portent sur les expérimentations didactiques menées par l'étudiant dans ses classes de stages. Enfin, l'analyse s'est faite plus fine: elle s'est centrée sur la proportion des savoirs, leur référencement, leur mode de référencialisation, le degré d'adhésion qu'ils suscitaient... Que révèle la cartographie des savoirs ainsi élaborée? De quels savoirs font état les étudiants? Quels paradigmes dominent? Quels référents théoriques sont objectivés, subjectivés, discutés, exhibés, illustrés ? En creux, que révèlent-ils des discours tenus par les formateurs d'enseignants ou les praticiens? Les pages qui suivent sont consacrées aux résultats de l'étude entreprise et à leur discussion. Des extraits tirés de différents TFE viendront illustrer et valider les constats posés. Un TFE en particulier, consacré à 
l'écriture du journal intime en tant qu'outil susceptible d'aider l'élève à construire son identité, nous servira de fil rouge : il s'avère en effet particulièrement exemplatif des résultats repérés dans le corpus.

\section{Les savoirs au cœur des TFE}

\subsection{Les savoirs scientifiques : des savoirs exhibés et des savoirs absents}

Les savoirs scientifiques récents relatifs à l'écriture sont tout à fait foisonnants, quelle que soit leur nature, leur champ épistémologique. Il serait vain d'en dresser ici un panorama exhaustif. Je vais me borner à esquisser quelques lignes de force des multiples recherches qui furent consacrées à la didactique de l'écrit dans le champ francophone. Plus spécifiquement encore, l'inventaire s'axera surtout sur les savoirs relatifs à l'enseignement-apprentissage de l'écriture au collège. En effet, de toute évidence, depuis une bonne trentaine d'années, les recherches didactiques consacrées à la production d'écrits au niveau de la scolarité obligatoire se sont multipliées. C. Barré-De Miniac affirme que si les années 70 furent les années "lecture », les années 80 furent les années "écriture ». Prises ensemble, elles semblent constituer un faisceau plus ou moins convergent, prises séparément, chacune d'elles représente le prisme spécifique d'un objet hétérogène. En effet, c'est un angle particulier de l'activité scripturale ou du scripteur qui est souvent mis en lumière. Quelques références possèdent toutefois un caractère plus généraliste. Je pense tout d'abord à l'ouvrage d'Y. Reuter (2000), qui me semble constituer une référence incontournable pour qui veut faire le point sur les connaissances en lien avec la didactique de l'écrit. Je pense également aux chantiers d'écriture popularisés par J. Jolibert et son Groupe d'Ecouen (2007 pour l'édition la plus récente), une référence déjà ancienne, mais qui a fait florès. L'écriture au collège a donné lieu notamment à deux ouvrages emblématiques (Barré-De Miniac \& Reuter, 2006 ; Bucheton \& Bautier, 1997). Ce à quoi s'ajoutent certains numéros de revues bien connues : les numéros 170, 174 et 181 du Français aujourd'hui (2010, 2011 et 2013), les numéros 26, 29, 49, 75 et 115-116 de Pratiques (respectivement 1980, 1981, 1986, 1992, 2002), les numéros 26 et 27 de Repères $(2002,2003)$. Par ailleurs, des ouvrages introductifs à la didactique du français accordent une large place à la didactique de l'écrit, dont ils permettent de saisir les principales tendances : J. David et S. Plane (1996), J.-L. Chiss et al. (2009), C. Simard (2010). L'excellente revue de vulgarisation Sciences humaines a consacré plusieurs dossiers conséquents à l'écriture, en octobre 2000, en été 2008 et, tout récemment, en novembre 2013. Enfin, l'engouement suscité par la diffusion des écrits de L. Vygotski (1934-1997) constitue la toile de fond de bon nombre de recherches dévolues à l'écrit.

Plusieurs recherches prennent en compte la problématique du scripteur, du sujet : il n'est qu'à voir le numéro 64 d'Enjeux (hiver 2005), le numéro 34 de Repères (2006), le numéro 45 de Recherches, le numéro 157 du Français aujourd'hui... D. Bucheton et É. Bautier (1997) analysent la figure du sujet écrivant, J.-C. Chabanne et D. Bucheton dirigent un ouvrage qui fait la part belle au concept de posture (2002), C. Tauveron et P. Sève (2005) préconisent quant à eux l'adoption d'une posture d'auteur par les jeunes élèves. La question du rapport noué par l'individu à l'écriture suscite d'autres recherches : C. BarréDe Miniac (2000), R. Delamotte-Legrand et al. (2000), Pratiques n 113-114 (2002), S.G. Chartrand \& C. Blaser (2008). M.-C. Penloup (1999) met au jour les pratiques extra- 
scolaires des collégiens et leur possible didactisation, ce qui a donné lieu à d'autres recherches, entre autres, le numéro 23 de Repères (2001) et le numéro 94 de la revue du Girsef (Leporcq, 2013). Le journal de lecture, le cahier d'écrivain, l'anthologie d'œuvres lues sont étudiés par M. Lebrun (2004). La question du biographique fait l'objet du numéro 147 du Français aujourd'hui. J.-L. Dumortier et al. (2009) envisagent l'écriture de soi à l'école. Les écrits réflexifs produits par les élèves donnent lieu à plusieurs publications (parmi lesquelles Crinon, 2000, 2002, 2008 ; Chabanne \& Bucheton, 2002 ; Scheepers, 2008, 2012). Delamotte-Legrand et Penloup (1994) utilisent le journal de bord pour faciliter les laborieuses tentatives de réécriture des collégiens. Penloup (1999) propose par ailleurs diverses activités centrées autour du journal intime pour les collégiens. S. Terwagne et al. (2001) expérimentent dans le primaire le « cahier de semences » : ce journal, qui peut être dialogué, accompagne la lecture d'ouvrages littéraires et permet de nourrir les débats qui se déroulent durant les cercles de lecture. Lebrun (1996) relate des expériences menées au collège de journal dialogué de lecture, envisagé comme un outil d'appropriation du texte littéraire. Quant à Lebrun (2004), elle évoque, pour le primaire, le journal de lecture, « journal d'apprentissage du débat interprétatif où le seul sujet à débattre est celui des lectures partagées ou à partager ». Le concept de littéracie renouvelle largement les études consacrées à l'écrit, comme le montrent notamment les numéros 131-132 et 153-154 de Pratiques (2006, 2012). La question du sujet scripteur s'est également articulée à celle de communauté discursive, étudiée par des chercheurs comme J.P. Bernié (2002), Jaubert et Rebière (2010).

Les opérations psycholinguistiques activées par l'écriture mobilisent d'autres chercheurs : J.-P. Bronckart $(1985,1996)$, B. Schneuwly (1988), C. Golder $(1993,1996)$... En particulier, les processus métacognitifs, mettant en jeu l'écriture scolaire ou non, retiennent des cognitivistes: F.-P. Büchel (1995), B. Noël (1997), P. Vianin (1999), P.A. Doudin et al. (1999). D'autres recherches s'affilient à la sociologie. Dans une perspective sociolinguistique, É. Bautier (1995) a montré la nécessité qu'il y avait à envisager les pratiques langagières des élèves, donc leurs tentatives effectives, appréhendées non pas en termes de carences linguistiques, mais susceptibles d'une analyse plurielle et compréhensive. Les travaux de B. Lahire $(1993,2008)$ traitent quant à eux de la culture scolaire écrite. Pour leur part, É. Bautier et J.-Y. Rochex (2008) élucident la question de la massification/démocratisation du lycée, l'écriture jouant un rôle non négligeable dans ce processus. Les dimensions socioculturelles propres à l'enseignement du français, donc aussi de l'écriture, sont au cœur d'autres investigations (Repères, $n^{\circ} 38,2008$ ).

L'écriture apparait également dans sa fonction de scolarisation des individus: il s'agit notamment d'envisager le binôme "écrire pour apprendre ", la perspective se voulant interdisciplinaire ou non, comme le montrent ces publications : G. Ducancel et J.-P. Astolfi (1995), J. Crinon (2000), I. Delcambre, J. Dolz et C. Simard (2000), É. Bautier (2001), J.C. Chabanne et D. Bucheton (2002), C. Barré-De Miniac et Y. Reuter (2006)... Par ailleurs, P. Schneeberger et A. Vérin (2009) interrogent les pratiques scripturales en sciences (2009). Bautier (2001) montre le lien qui existe entre le langage, dont le langage écrit, et la scolarisation: s'appuyant sur de nombreux travaux, elle met au jour le rapport étroit entre un usage littéracié du langage et la réussite scolaire. Dès lors, l'écriture à l'école est investie de multiples fonctions, heuristiques ou sélectives.

16 L'écriture de fiction a donné lieu à nombre de travaux, je pense notamment à J.-L. Dufays et S. Plane (2009), à C. Tauveron et P. Sève (2005), aux numéros 48, 83, 127, 133-134 de la revue Pratiques (1985, 1994, 2002, 2007), au numéro 153 du Français aujourd'hui (2006), aux 
numéros 13, 33 et 40 de la revue Repères (1996, 2006, 2009). S'agissant expressément de l'atelier d'écriture, l'ouvrage publié par O. Pimet et C. Boniface en 1999 reste une référence incontournable, tout comme le numéro 61 de Pratiques (1989) ou le livre de . Lafont-Terranova (2009).

D'autres recherches encore prennent en compte une dimension spécifique de l'écriture telle qu'elle se déploie à l'école. Je pense d'abord à la réécriture, placée à l'aune de la génétique textuelle: A. Séguy (1994), D. Bucheton (1995), J. David (2001), D. Bessonnat (2001), C. Fabre-Cols (2002)... Ce à quoi il faut ajouter le numéro 144 du Français aujourd'hui (2004), le numéro 55 de Recherches (2011), les numéros 105-106 de Pratiques (2000), le numéro 10 de Repères (1994), parmi lesquels il faut compter notamment les travaux de C. Boré. L'orthographe a fait l'objet de maintes publications, le lecteur pourra se reporter notamment aux numéros 25 et 46 de Pratiques (1979 et 1985), aux ouvrages plus récents de J.-P. Jaffré (1992), de D. Manesse et D. Cogis (2007) ou encore de M. Fayol et J.-P. Jaffré (2008)... Quant au lexique, il n'est pas en reste, mentionnons ces deux références très actuelles, les numéros 155 et 156 de la revue Pratiques (2012), sans oublier l'ouvrage édité par C. Garcia-Debanc et al. en 2013. Les pratiques scripturales numériques suscitent un vif engouement, comme en témoigne le récent colloque de l'AIRDF (Association internationale de recherche en didactique du français) qui s'est tenu à Lausanne en août 2013. Le lecteur pourra utilement se référer, entre autres, à G.-L. Baron et al. (2005), N. Marty (2005), C. Fluckiger (2008), M.-L. Elalouf (2012)... En particulier, l'argumentation retient l'attention des chercheurs, il n'est qu'à voir la récurrence des numéros thématiques que Pratiques lui consacre : numéros 28 (1980), 68 (1990), 73 (1992), 84 (1994), 96 (1997). Le Français aujourd'hui a consacré ses numéros 123 et 145 à cette problématique, en 1998 et en 2004. Il faut y adjoindre d'autres publications: B. Schneuwly (1988), C. Golder $(1993,1996)$, I. Delcambre (1997), B. Schneuwly et J. Dolz (2006), J.-L. Dumortier et al. (2011), C. Scheepers (2013).

La rapide et non exhaustive évocation qui précède montre que les chercheurs en didactique se sont maintes et maintes fois évertués à cerner l'écriture scolaire sous de multiples facettes. Certaines références sont plus pointues, d'autres s'adressent plus explicitement aux (futurs) enseignants. Dès lors, lesquelles de ces références viennent étayer les 47 TFE qui constituent mon corpus? Si j'avais postulé un décalage entre le foisonnement des recherches en matière de didactique de l'écrit et les sources convoquées par les futurs enseignants, je ne devinais pas quelle était l'ampleur de ce décalage... En effet, de toute évidence, les étudiants ne mobilisent que très exceptionnellement les didacticiens de l'écrit dans le cadre de leur recherche. Si l'écriture est omniprésente dans les réflexions ou les pratiques de classe, elle n'est généralement pas conceptualisée, pensée, discutée, réfléchie, étayée, interprétée au moyen des savoirs produits par les chercheurs en didactique de l'écrit. Ainsi, l'ouvrage de Pimet et Boniface (1999) est cité par trois étudiants. Sont mentionnés à une seule reprise des ouvrages pourtant généralistes, clairs, didactisables comme celui de Reuter (2000) ou de Jolibert (2007). Sont mentionnés à raison d'occurrences isolées les travaux de Chervel, Olson, Lahire, Bucheton, Crinon, Scheepers, Manesse et Cogis, Tauveron, Dezutter, Collès, Charmeux... Les revues n'ont guère plus de succès: la revue Pratiques est convoquée à deux reprises, la revue Le français aujourd'hui sept fois (dont six par le même étudiant !), la revue Enjeux dix fois (dont six par un seul étudiant...). Même les manuels, souvent conçus par les didacticiens, ne sont que très ponctuellement évoqués. 
19 La bibliographie reproduite en annexe est tirée du TFE dont le titre est : Le journal intime peut-il aider l'élève à construire son identité ? Elle renvoie vers trois sites internet, quatre ouvrages (un essai et trois livres scientifiques) et deux articles tirés de la revue Sciences humaines, revue de vulgarisation. De façon générale, la bibliographie parait donc assez mince. Il faut en outre constater que si les références mentionnées s'attachent à clarifier la problématique de l'identité, de l'adolescence ou du journal intime, aucune ne relève du champ de la didactique du français ou de la didactique de l'écriture en particulier. Dès lors, les activités scripturales proposées en classe ne sont pas étayées par des garants scientifiques.

Dans ce TFE comme dans beaucoup d'autres, tout semble se passer comme si la pratique scripturale allait de soi. L'écriture ne doit pas être définie, l'enseignement-apprentissage de l'écriture ne doit pas être conceptualisé, théorisé, fondé scientifiquement, légitimé par des garants scientifiques, référé à des pratiques didactiques imaginées et diffusées par d'autres... Pourtant, les dispositifs des étudiants reposent sur des principes relativement immuables : les activités lecturales précèdent et préparent les activités scripturales, les bains de textes permettent aux élèves de mieux écrire, les consignes doivent être attractives, il faut créer de la motivation, les élèves n'aiment pas forcément écrire, les ateliers d'écriture constituent des outils incontournables, la créativité nait de la contrainte, l'écriture s'accomplit en plusieurs jets, il faut socialiser les textes, il ne faut pas toujours se soucier de l'orthographe, l'écriture est une compétence transversale qui peut être mise au service de bien d'autres compétences... Bref, le discours se fait volontiers injonctif, mais sans que les prescriptions énoncées ou laissées largement implicites ne soient justifiées, validées en aucune façon, comme le montre ce bref extrait : «Il est nécessaire que le scripteur prenne conscience qu'écrire, c'est aussi et surtout planifier, relire et réécrire ». Le lecteur averti reconnaitra les propos de J. R. Hayes et L. S. Flower, mais ni eux ni ceux qui les ont diffusés dans le champ francophone ne sont mentionnés. Les principes implicites sous-jacents aux dispositifs didactiques s'apparentent à des dogmes non discutés, non interrogés, non situés, non argumentés. Faut-il y déceler les doxas didactiques en vigueur dans les hautes écoles? Rares sont les passages où les étudiants font interagir leurs propres constats et les travaux des didacticiens. C'est le cas dans cet extrait : « Pour avoir beaucoup lu des écrits publiés par Jacques Crinon et Caroline Scheepers, notamment autour de la mise en place de différents journaux au service de la métacognition, j'étais déjà convaincue de la pertinence et de la richesse de cet outil. Mais je dois vous l'avouer, les résultats de mon expérimentation ont dépassé ce que j'avais envisagé. En effet, je peux vraiment parler de bénéfices inattendus ». Suit une comparaison entre les résultats identifiés par l'étudiante et ceux relevés par les chercheurs.

21 Il parait clair que quand le TFE se fait davantage documenté, les savoirs expérienciels qui sont énoncés paraissent plus formalisés, plus fins, plus précis, plus jargonnants : «Il faut aussi mettre en évidence que les élèves se sont rendu compte qu'ils pouvaient agir sur l'écriture sans se laisser dominer par elle. Ils ont clairement perçu que l'écrit pouvait avoir des utilités et des fonctions diverses. En retravaillant leur premier jet et en réfléchissant à leurs démarches d'écriture, ils se sont construit une représentation dynamique de l'écriture ». Étonnamment, la didactique de la lecture est bien plus souvent convoquée au cœur de TFE pourtant dévolus à l'écriture et ce, autour de quelques figuresphares comme Giasson, Dufays, Terwagne, Vanhulle et Lafontaine, Poslaniec, Lafontaine... 

matière d'écrit? Peut-être les étudiants ont-ils tendance à naturaliser l'activité scripturale, pensant que faire écrire constitue une tâche simple, laquelle consiste à inviter les élèves à prendre une feuille et un crayon? Dans une recherche précédente, j'ai pu montrer (Scheepers, 2014) que les planifications de leçons axées sur l'écriture font généralement l'objet de préparations très brèves, parfois inexistantes, les étudiants semblant penser que faire écrire ne requiert aucun dispositif à planifier, ne sollicitant pas de consigne détaillée, de savoirs préexistants, de réajustements en cas de difficultés... Alors qu'une séquence visant à faire acquérir des compétences grammaticales ou orthographiques sera préparée dans ses moindres détails. Tout se passe comme si les futurs enseignants estiment que faire écrire les apprenants ne s'apparente pas à un «travail » de leur part, ne relève pas d'une quelconque ingénierie didactique, sur le mode $\mathrm{du}$ « je ne donne pas cours, je fais écrire les élèves». Bref, je ne suis pas au centre de l'activité, je suis en retrait. Se pose ici avec acuité toute la conception dont font état les futurs enseignants à propos de l'écriture et de son enseignement-apprentissage. Quelle est leur représentation de l'écriture et des tâches didactiques qui y sont liées? Une autre hypothèse interprétative réside naturellement dans l'éventuelle méconnaissance dont semblent faire montre les étudiants (et certains de leurs formateurs?) quant aux acquis générés par la didactique de l'écrit, laquelle permet selon moi largement d'éclairer, de fonder, d'interpréter et de renouveler les pratiques didactiques en classe. Les dispositifs d'écriture semblent relever du bricolage, chacun « réinventant la roue » dans son coin, quand des travaux clairs, compréhensibles et accessibles pourraient constituer un solide appui au service de la pratique. Sans doute faut-il le regretter et se questionner : que font les chercheurs pour diffuser les résultats de leurs travaux au-delà de leur cercle restreint? Comment faire davantage circuler les savoirs entre les différentes sphères concernées : chercheurs, inspecteurs, directions, formateurs d'enseignants, enseignants, futurs enseignants, décideurs politiques, formateurs... de formateurs?

Concernant les savoirs scientifiques disciplinaires, donc les savoirs liés aux objets à enseigner, leur présence dans les bibliographies est nettement plus massive. En particulier, les savoirs linguistiques prédominent. De grandes figures de linguistes sont mentionnées: Blampain, Goosse, Klinkenberg, Wilmet, Catach, Grevisse, Benveniste, Bentolila, Amossy, Maingueneau, Walter, Jakobson... Du côté littéraire, les ouvrages de Lejeune sont abondamment évoqués, dès lors que le TFE concerne l'autobiographie, le journal intime, le blog... Barthes ou le mouvement Oulipo sont cités à quelques reprises. Les étudiants font généralement état de solides connaissances en matière de littérature de jeunesse. Il arrive que des philosophes soient convoqués, parmi lesquels Platon, Aristote, saint Augustin, Kant, Fichte, Husserl, Sartre... De façon générale, les thématiques qui font l'objet du TFE donnent lieu à des bibliographies plutôt étoffées et pertinentes, qu'il s'agisse de conceptualiser à propos des émotions, de la Shoah, de la publicité, du langage SMS ou du slam. Le décalage entre ce souci de recherche documentaire à propos des thématiques traitées accentue encore la raréfaction des théories sur l'enseignementapprentissage de l'écriture. Faut-il tisser un lien entre ce constat et le point de vue exprimé par H.-E. Tenorth (2009) selon lequel les enseignants du secondaire sont bien plus soucieux des contenus académiques que de leur transposition didactique? Leur érudition porterait sur les objets à enseigner, non sur leur didactisation. S'agit-il d'une forme de mépris pour la didactique, jugée peu noble et peu utile ? D'une forme d'élitisme qui permettrait de se distinguer des instituteurs, à la culture plus didactique? D'un

Pratiques, 161-162 | 2014 
reliquat des années passées à l'université ? De la considération qui voudrait qu'au fur et à mesure que l'on progresse dans la scolarité, la complexité des savoirs primerait sur leur mode d'enseignement?

Cela étant, il me faut nuancer quelque peu le propos : d'une part, un très grand nombre de références bibliographiques savantes listées dans la bibliographie ne sont jamais mentionnées au cœur du TFE lui-même, d'autre part, beaucoup de sources convoquées au fil du TFE ne sont pas répertoriées dans la bibliographie... Même si nous n'avons pas affaire à des travaux universitaires, ce manque de rigueur et de cohérence questionne à tout le moins. Tout comme interpelle la récurrence des passages non clairement référencés : beaucoup de développements ne sont clairement pas imputables à l'auteur du TFE, mais ne sont pas pour autant associés à une source bibliographique. En revanche, un lecteur averti pourra reconnaitre les traces de tel ou tel ouvrage, non mentionné en tant que tel... Des cas avérés de plagiat sont donc décelables. Par conséquent, la littérature mentionnée dans la bibliographie parait parfois purement ornementale: comme si sa seule présence servait à cautionner le travail entrepris. Les savoirs paraissent exhibés, mais non fonctionnalisés, puisque les théories sont répertoriées dans la bibliographie, mais ne viennent pas étayer la recherche menée. Ailleurs, il arrive que des chapitres entiers soient précédés d'une mention globalisante de l'ordre: «Les informations qui suivent sont inspirées de telle source». Dès lors, le TFE prend l'allure d'un vaste patchwork. Certains étudiants mentionnent par exemple les œuvres de Darwin ou de Descartes: il s'agit vraisemblablement de citations de seconde main, mais elles ne sont pas mentionnées comme telles. Au fil des années, s'accroit sensiblement la part réservée aux informations glanées sur l'internet: dans un TFE, une étudiante reprend dans sa bibliographie deux seuls ouvrages et pas moins de 18 sites internet. De nouveau, le TFE consacré à l'écriture du journal intime donne à voir les constats émis. Ainsi, la page 17, reproduite en annexe constitue l'amorce d'un chapitre dans lequel l'étudiante s'attache à définir le genre diaristique. En particulier, elle retrace l'historique du journal intime, de son apparition à son évolution au fil des siècles. De toute évidence, les propos ne sont pas imputables à l'étudiante : sans doute les a-t-elle, au mieux, reformulés au départ d'une source bien informée dont les références ne sont pas communiquées. En revanche, elle évoque différents auteurs littéraires très réputés, de saint Augustin à Constant, et les références bibliographiques de leurs journaux intimes, lesquelles ne figurent pas dans la bibliographie. Corbin et Perrot, auteurs d'ouvrages scientifiques, sont mentionnés et reformulés, mais l'on ne trouve nulle trace à leur sujet dans la bibliographie. Par contre, les sources répertoriées dans la bibliographie qui sont dévolues au journal intime et d'où pourraient provenir les informations recensées ne sont pas convoquées dans cette page.

Quant aux savoirs pédagogiques, des constats relativement similaires pourraient être établis. Une figure comme Meirieu est massivement citée, tout comme Piaget, Stordeur ou Freud. En revanche, Vygotski est très peu mentionné, tout comme Crahay, De Vecchi, Caron, Perrenoud, Charlot, Viau ou même Freinet... Les étudiants font état à plusieurs reprises d'articles parus dans la revue Les Cahiers pédagogiques, La Revue française de pédagogie est, elle, bien moins citée.

\subsection{Les savoirs curriculaires : des savoirs valorisés}

L'examen des savoirs curriculaires montre sans nul doute possible que les savoirs véhiculés au cours du cursus académique sont largement valorisés par les étudiants. En 
atteste particulièrement cet extrait emblématique : «En première année à la haute école, nous avons vu et étudié dans le cadre du cours de FLE un tableau élaboré par M.X concernant la construction des temps simples de la conjugaison. Ce fut comme un déclic pour moi ». Cet autre passage illustre également ce phénomène : «Parmi les lectures que j'ai pu réaliser au cours de mes deux premières années d'études, un livre a plus particulièrement attiré mon attention et fut pour moi d'une importance capitale. L'apprentissage de l'abstraction de Britt-Mari Barth. Ce sujet m'a passionnée et j'ai éprouvé l'envie de réaliser un TFE en lien avec les capacités d'abstraction ». Tout se passe dans les TFE comme si le parcours de formation à la haute école était foncièrement survalorisé. Pourquoi ? Peut-être faut-il se rappeler que le TFE s'inscrit dans un cadre certificatif, certains étudiants se montrant dès lors soucieux de ne pas indisposer leurs lecteurs... Très régulièrement, les étudiants s'appuient sur des notes de cours (il est vrai immédiatement disponibles et supposées vulgarisantes), soulignent les moments-clés de leur cursus... Dans ce portrait euphorique, la formation s'apparente à un vaste et pertinent répertoire de dispositifs, de cadres conceptuels, de pistes interprétatives... En outre, abondent les doxas et injonctions propres aux formations initiales d'enseignants : il faut rendre l'élève acteur de ses apprentissages, il faut donner du sens aux apprentissages, il faut donner des contraintes aux élèves pour les faire écrire... Les propos enthousiastes relatifs au cursus académique qui émaillent les TFE contrastent singulièrement avec les réflexions souvent très critiques dont font généralement état les étudiants au sujet de leur formation, dans des contextes, il est vrai, beaucoup moins formels et moins porteurs d'enjeux... Enfin, plusieurs étudiants mentionnent leurs notes de cours universitaires, vestiges manifestes de leur passage à l'université, ce qui renforce le constat selon lequel l'inscription à la haute école relève souvent d'un second choix pour ce public.

Rares sont les études qui se sont penchées sur le curriculum des formations initiales d'enseignants. Dans le cadre d'un mémoire de master, L. Caprace (2011) a analysé les curriculums de formation en français en vigueur pour les futurs instituteurs du primaire. Son analyse est sévère et elle relève des incohérences graves entre différents établissements, au sein d'un même établissement. À ma connaissance, une recherche similaire relative à la formation des futurs agrégés reste à faire, mais il est clair que l'analyse des TFE suggère des questionnements cruciaux du point de vue de la formation des enseignants ou de la formation des formateurs.

\subsection{Les savoirs décrétaux : des savoirs incontournables}

Tout comme les savoirs curriculaires, les savoirs décrétaux ont une présence bien marquée dans les bibliographies. Rares sont les TFE qui ne mentionnent pas les textes légaux prescriptifs que sont les Socles de compétences ou les Compétences terminales, lesquels spécifient les compétences qui doivent être atteintes au terme d'un cycle d'apprentissages, ou les programmes d'enseignement, propres à un réseau (officiel, libre...) et stipulant les démarches susceptibles de soutenir le développement des compétences attendues. Issus de larges concertations au sein d'équipes hétérogènes, ces documents s'appuient de toute évidence sur les acquis les plus récents de la recherche scientifique, sur les dimensions qui semblent faire l'objet d'un large consensus. Ainsi, une étudiante recopie de longs passages d'un programme qui reformule de toute évidence les travaux de Penloup (1999) et en souligne tout l'intérêt... Dès lors, les savoirs didactiques 
qui font cruellement défaut dans les TFE s'insinuent indirectement par le truchement des textes officiels prescrivant les pratiques didactiques à mettre en place. Cependant, il est fréquent que ces mêmes textes soient consciencieusement listés dans la bibliographie, mais ne soient pas mis au service de la démonstration construite par l'étudiante, lorsqu'il s'agit notamment de recenser les compétences visées par une activité. Dans certains cas, il arrive que les étudiants se montrent critiques face aux savoirs décrétaux : «Il y a peu de traces des émotions dans les programmes. [...]. C'est à mon sens assez regrettable ».

\subsection{Les savoirs expérienciels : des savoirs euphoriques, mais peu étayés}

Les savoirs que les étudiants se forgent au départ de leurs pratiques didactiques s'avèrent très généralement euphoriques, complaisants, enthousiastes : «Je suis assez satisfait du travail réalisé en si peu de temps! Je pense que les élèves ont retiré beaucoup de choses. [...] Cette leçon a bien fonctionné car elle était basée sur la discussion [...]. Et c'est vrai que c'était bien! » Un autre extrait de cet ordre : «Cette expérience était unique pour moi, a répondu à mes attentes et m'a procuré énormément de plaisir ». De plus, il est fréquent que les constats posés par les étudiants soient à la fois lapidaires et peu argumentés, un étudiant se contentant par exemple d'un : «Cette activité a assez bien fonctionné », sans prendre la peine d'étayer son propos. D'ailleurs, globalement, la présence des élèves, donc des scripteurs, dans les TFE s'avère tout à fait fantomatique: ils sont très peu individualisés, très peu caractérisés. Soit leurs écrits sont évoqués allusivement, sur le mode réjoui du " ça a bien marché ! », soit leurs écrits sont reproduits en annexe, mais ils ne font pas davantage l'objet d'interprétations fines, d'évaluation circonstanciées, de liens avec les savoirs savants, de toute façon souvent inexistants. Ces observations rejoignent celles avancées par d'autres chercheurs, en l'occurrence É. Nonnon (1995), M. Guigue et J. Crinon (2003), lesquels constatent que les élèves ont généralement bien peu d'épaisseur dans les mémoires professionnels. La plupart du temps, la description de l'activité prend nettement le pas sur son interprétation, laquelle se réduit souvent à très peu de choses. Ainsi, un long dispositif centré sur un atelier d'écriture donne lieu à ce seul commentaire, très vague: "Les élèves peuvent s'approprier une consigne de différentes manières. Cela dépend de leur compréhension, de ce qu'ils ont envie d'écrire et de partager, mais aussi de la façon dont ils "déchiffrent" le support imagé ». Mais quels sont les modes d'appropriation adoptés par les apprenants? Quelles conduites scripturales peut-on identifier?

Lorsque les appréciations sont plus nuancées, le temps est fréquemment invoqué comme un facteur explicatif :

"J'ai été assez déçue que cette leçon soit la dernière étape de lecture, car l'exploitation n'a pas pu être aussi complète que ce que j'en attendais. Le timing a été un véritable adversaire pour cette activité ». Un autre commentaire similaire : «Ce qui me semble le plus positif à retirer de cette expérimentation est que les élèves semblent avoir acquis après pourtant si peu de temps, quelques réflexes de relecture et surtout de questionnement lorsqu'ils écrivent. [...] La méthode choisie [...] a été globalement concluante. [...] Bien que mes objectifs de départ aient pu être respectés étant donné que j'ai dû m'adapter au peu de temps dont j'ai pu disposer, il me semble que l'expérimentation a quand même apporté quelque chose aux élèves ». 
31

évidence, la conduite argumentative pose ici question : qu' ont appris exactem les élèves ? En quoi la méthode s'est-elle avérée " globalement concluante »? En quoi les objectifs ont été respectés ? Quelle est la nature du " quelque chose » apporté aux élèves? En outre, il me semble que l'invocation régulière du manque de temps permet à peu de frais de se montrer critique face à l'expérimentation menée : nous avons affaire à une causalité externe, l'étudiant imputant les éventuelles failles de son dispositif au temps limité que lui a concédé son maitre de stage. générées chez les élèves que sur des facteurs d'ordre motivationnel ou affectif : "La première constatation concernant ce dispositif a été le regain de motivation de la part des élèves ", " ces activités furent très appréciées ", " je pense que la clé de ce succès provient prioritairement de la mise en confiance établie dès le départ ", " cette expérience était unique pour moi, a répondu à mes attentes et m'a procuré énormément de plaisir », " prendre connaissance des écrits d'un élève permet au professeur de prendre conscience de l'état émotionnel de cet élève "... Tout se passe dès lors comme si le seul critère d'évaluation relevait des sensations, des sentiments... À d'autres moments, les doxas curriculaires resurgissent: "Afin que les élèves entrent facilement dans cette tâche, j'ai décidé de commencer par une activité assez ludique ", "d'où l'importance de conférer sans cesse du sens aux exercices ».

Enfin, il arrive que les étudiants pointent eux-mêmes les carences dont souffre leur dispositif, carences qui auraient de toute évidence pu être évitées si les tâches scripturales proposées aux apprenants avaient été davantage planifiées et conceptualisées :

"Je suis mitigée quant aux résultats de cet exercice. Les élèves, amusés par le travail de création de groupe, ont un peu oublié qu'il s'agissait, avant tout, d'un travail et non d'une activité exclusivement ludique. De plus, les sujets choisis étant tellement vastes, les élèves ne savaient pas dans quelle direction ils devaient aller. Le genre de texte devant être écrit à partir de ce sujet n'avait pas été expliqué clairement, ce qui a, pour certains textes donnés [sic) des résultats non escomptés ».

Cet autre extrait suggère les mêmes conclusions : "J'avais oublié de préciser qu'il fallait d'abord faire un brouillon et certains étudiants ont remis un travail peu soigné (ratures, traces de crayon mal gommées...) ». Il me semble pouvoir avancer l'hypothèse selon laquelle si les activités didactiques se fondaient davantage sur des savoirs scientifiques, les tâches seraient plus pertinentes, les analyses plus fines. Les futurs enseignants se questionneraient peut-être davantage sur les apprentissages accomplis par les élèves plutôt que sur les sentiments suscités par les tâches. Dit autrement, les savoirs permettraient d'étayer la secondarisation des pratiques enseignantes et apprenantes.

\section{Conclusion}

La question qui s'est inscrite au cœur de ma contribution était la suivante : quels savoirs d'ordre scientifique, curriculaire, décrétal et expérienciel sont mobilisés par les futurs agrégés dans le travail de fin d'études qui clôture leur formation initiale en haute école? Ainsi, nous avons considéré que le TFE s'envisage dans cette perspective comme un observatoire et un moteur privilégiés des savoirs qu'est supposé convoquer et façonner l'apprenti-enseignant pour concevoir, expérimenter, accompagner, réguler et évaluer un 
dispositif d'écriture destiné aux élèves du début du secondaire. Le TFE, équivalent du mémoire professionnel, constitue à mes yeux ce que j'ai appelé un observatoire privilégié, car il suppose l'intrication étroite de connaissances, concepts, représentations ou paradigmes avec des données empiriques hétérogènes. Le signataire du travail de fin d'études adopte une posture triple et ambiguë : encore étudiant, il doit pourtant faire valoir d'authentiques compétences et composantes identitaires professionnelles, tout en prenant en compte un objet avec lequel il a peut-être noué un investissement affectif fort. En outre, le scripteur élabore son projet de recherche sous la supervision plus ou moins étroite d'un promoteur, en l'occurrence un formateur de la haute école, chargé au terme du processus d'évaluer le travail fourni. Le dispositif institué par l'apprenti-enseignant s'inscrit de plus dans un contexte éducatif spécifique, réunissant élèves, maitres de stage, praticiens, direction, inspection, parents... Par conséquent, le TFE s'avère éminemment polyphonique, puisqu'y sont tissées les voix des chercheurs et des acteurs de terrain.

Au terme de l'examen de mon corpus, non exhaustif et non représentatif, insistons-y, je serais bien en peine de déterminer les modèles didactiques dominants dans les TFE dont j'ai pu prendre connaissance, tant les références de ce point de vue sont rares, voire absentes. Il faut cependant se garder d'en déduire que l'écriture ne tient pas une place importante dans les plans de formation, mais il faut se borner à constater que les savoirs, en particulier les savoirs didactiques, sur lesquels les étudiants étayent leurs pratiques de classe paraissent bien minces, souvent inexistants. Et ce, en dépit de l'extrême vitalité des recherches en la matière ces dernières années. Il appert qu'est réellement considérable le décalage entre les travaux scientifiques menés et ceux qui sont mobilisés par les futurs enseignants dans leur TFE. Or, si l'on considère avec Hofstetter et Schneuwly (2009) que les savoirs sont centraux dans la formation initiale des enseignants, si l'on admet que le TFE constitue le genre académique par excellence dans lequel les étudiants doivent mobiliser des savoirs scientifiques, il me semble alors que le constat s'avère éminemment problématique.

Si l'écriture est centrale dans les pratiques scolaires, elle ne parait pas réellement conceptualisée, pensée, discutée, réfléchie. Même des ouvrages généralistes et accessibles comme ceux de Reuter (2000) ou de Jolibert (2007) ne sont guère mentionnés par les étudiants. Les résultats que l'analyse des TFE suggère conduisent à se questionner sur les représentations des futurs enseignants en matière d'écriture et d'enseignementapprentissage de l'écriture. Qu'est-ce que l'écriture pour eux? Que pensent-ils de sa didactisation? De plus, il semble bien que les étudiants s'affilient à autant de principes didactiques similaires (les bains de textes, la socialisation des écrits, etc.), mais ces derniers ne sont guère validés par des études scientifiques, ils fonctionnent par conséquent comme des doxas. Il faut en outre noter que la didactique de la lecture parait bien mieux documentée, y compris dans les TFE dévolus à l'écriture.

La cartographie des savoirs décelables ou absents dans le corpus recueilli, bien que non exhaustif et non représentatif, conduit à formuler un bilan en demi-teintes et, partant, pourrait conduire à réinterroger la formation d'enseignants et la formation des formateurs. Quelques recherches longitudinales ont pu montrer le difficile processus de subjectivation des savoirs au fil d'une formation initiale d'enseignants : S. Vanhulle (2002, 2009), C. Scheepers (2009), A.Clerc (2013). Ces travaux montrent des trajectoires hétérogènes d'appropriation des savoirs qui s'enseignent au fil du cursus académique. Ils indiquent par ailleurs que des savoirs enseignés aux savoirs appris, la marge est parfois considérable. Ils mentionnent également des pistes susceptibles de soutenir une pleine 
subjectivation des savoirs, parmi lesquelles l'écriture réflexive, l'alternance théoriepratique, l'isomorphisme entre les dispositifs expérimentés en formation initiale et ceux à transposer au primaire ou au secondaire...

La cartographie établie invite en outre les chercheurs à se questionner quant à la diffusion de leurs travaux: en effet, l'étude ici conduite suggère que les "savoirs savants " fonctionnent trop souvent en vases clos, alors qu'ils devraient idéalement circuler d'une instance à l'autre. Pour ce faire, de multiples initiatives mériteraient d'être imaginées, puis tentées. Ainsi, de nouveaux supports de diffusion des travaux scientifiques restent à créer ou à promouvoir : revues vulgarisatrices, sites rigoureux, mais accessibles... Les recherches collaboratives pourraient être multipliées. Les carrières des chercheurs, des formateurs ou des académiques pourraient être jugées à l'aune d'autres critères que ceux actuellement en vigueur : tous pourraient être incités à diffuser les résultats de leurs travaux. Bien d'autres pistes pourraient être empruntées. L'enjeu est considérable : renforcer les interactions entre la recherche, la formation d'enseignants, la formation de formateurs et les classes me parait de nature à tisser des liens plus étroits entre savoirs savants et expérienciels. Or, ce tissage pourrait enrichir considérablement les pratiques et les représentations des uns et des autres, au service d'un enseignement plus juste et plus efficace, mais aussi dans l'optique d'une recherche plus soucieuse de son apport à la communauté.

\section{BIBLIOGRAPHIE}

BARON, G.-L. et al. (2005) : Le multimédia dans la classe à l'école primaire, Lyon, Institut national de recherche pédagogique.

BARRÉ-DE MINIAC, C. (1995) : «La didactique de l'écriture : nouveaux éclairages pluridisciplinaires et état de la recherche ", Revue française de pédagogie, 113, 1995, p. 93-133.

- (2000) : Le rapport à l'écriture. Aspects théoriques et didactiques, Lille, Presses universitaires du Septentrion.

BARRÉ-DE MINIAC, C. \& REUTER, Y. (2006) : Apprendre à écrire au collège dans les différentes disciplines, Paris, INRP.

BAUTIER, É. (1995) : Pratiques langagières, pratiques sociales. De la sociolinguistique à la sociologie du langage, Paris, Éd. L'Harmattan.

- (2001) : « Pratiques langagières et scolarisation », Revue française de pédagogie, 137, p. 117-161.

BAUTIER, É. \& Goigoux, R. (2004) : « Difficultés d'apprentissage, processus de secondarisation et pratiques enseignantes : une hypothèse relationnelle », Revue française de pédagogie, 148 , p. 89-100.

BAUTIER, É., ROCHEX, J.-Y. (1997) : Les « nouveaux Lycéens ». Massification ou démocratisation ?, Paris, A. Colin. 
BERNIÉ, J.-P. (2002) : « L'approche des pratiques langagières scolaires à travers la notion de "communauté discursive" : un apport à la didactique comparée ", Revue française de pédagogie, 141, p. 67-88.

BOCH, F. \& GROSSMANN, F. (coords) (2001) : « Apprendre à citer le discours d'autrui », Lidil, 24. BRISSAUD, C. \& BESSONNAT, D. (2001) : L'orthographe au collège. Pour une autre approche, Paris, Delagrave.

BRONCKART, J.-P. (1985) : Le fonctionnement des discours, Lausanne/Paris, Delachaux et Niestlé. - (1996) : Activité langagière, textes et discours, Lausanne/Paris, Delachaux et Niestlé.

BÜCHEL, (1995) : L'éducation cognitive, Lausanne, Delachaux et Niestlé.

BUCHETON, D. (1995) : «La didactique du français au carrefour des métiers d'enseignants, de formateurs et de chercheurs ", in : J.-L. Chiss et al. (dirs), La didactique du français, état d'une discipline, Paris, Nathan.

BUCHETON, D. \& BAUTIER, É. (1997) : Conduites d'écriture au collège et au lycée professionnel, Versailles, Centre régional de documentation pédagogique de l'académie de Versailles.

CAPRACE, L. (2011) : Les curriculums de formation en français à destination des futurs instituteurs primaires en Communauté française de Belgique, mémoire inédit, université de Liège.

CHABANNE, J.-C. \& BUCHETON, D. (dirs) (2002) : Parler et écrire pour penser, apprendre et se construire, Paris, Presses universitaires de France.

CHARTRAND, S.-G. \& BLASER, C. (dirs) (2008) : Le rapport à l'écrit : un outil pour enseigner de l'école à l'université, Diptyque, 12.

CHISs, J.-L. et al. (2009) : Didactique du français. Fondements d'une discipline, Bruxelles, De Boeck.

CLERC, A. (2013) : Rôle des savoirs théoriques de référence dans les parcours de formation des futurs enseignants des premiers degrés de la scolarité, thèse en sciences de l'éducation, université de Genève.

CRINON, J. (coord.) (2000) : « Dossier : écrire pour apprendre », Les Cahiers pédagogiques, 388-389. - (2002) : «Écrire le journal de ses apprentissages », in : J.-C. Chabanne \& D. Bucheton (dirs) Parler et écrire pour penser, apprendre et se construire, Paris, PUF, p. 123-143.

CRINON, J. \& GUIGUE, M. (2006) : «Écriture et professionnalisation », Revue française de pédagogie, 156, p. 117-169.

DAVID, J. (2001) : «Étude de la langue et lecture-compréhension : l'exemple du lexique », in: Observatoire national de la lecture, La Lecture de 8 à 11 ans, Paris, ministère de la Recherche/ ministère de l'Éducation nationale, p. 34-38.

DAVID, J. \& PLANE, S. (dirs) (1996) : L'apprentissage de l'écriture de l'école au collège, Paris, PUF. Delamotte-Legrand, R. et al. (2000) : Passages à l'écriture, Paris, PUF.

DelAmotTE-LEGRAND, R. \& PENLOUP, M.-C. (1994) : « Du journal de bord au collège comme lieu d'émergence des représentations de la réécriture », Repères, 10, p. 83-94.

DELCAMBRE, I. (1997) : L'exemplification dans les dissertations. Étude didactique des difficultés des élèves, Villeneuve d'Ascq, Presse universitaires du Septentrion.

Delcambre, I., Dolz, J. \& Simard, C. (2000) : «Écrire pour apprendre, une activité complexe aux sens multiples ", Lettre de l'AIRDF, 26, p. 3-5. 
De Nuchèze, V. (1998) : « Approche pragmatico-énonciative du discours de recherche », Lidil, 17, p. 25-40.

Doudin et al. (1999) : Métacognition et éducation, Berne, P. Lang.

DUCANCEL, G. \& ASTOLFI, J.-P. (coords) (1995) : « Apprentissages langagiers, apprentissages scientifiques ", Repères, 12.

DUFAYS, J.-L. \& PLANE, S. (dirs) (2009) : L'écriture de fiction en classe de français, Namur, Presses universitaires de Namur.

DUMORTIER, J.-L. (coord.) (2009) : « Pour aborder en classe l'écriture de soi », Tactiques, 5.

- (coord.) (2011) : « Pour le discours incitatif », Repères, 34.

ELALOUF, M.-L. (dir.) (2012) : « Les connaissances cachées développées par la lecture et l'écriture électronique extrascolaires ", ELA. Études de linguistique appliquée, 165.

Enjeux (2005) : 64, « La question du sujet».

FABRE-COLS, C. (2002) : Réécrire à l'école et au collège, Paris, ESF.

FAYOL, M. \& JAFFRÉ, J.-P. (2008) : Orthographier, Paris, PUF.

FLUCKIGER, C. (2008) : «L'école à l'épreuve de la culture numérique des élèves ", Revue française de pédagogie, 163, p. 51-61.

GARCIA-DEBANC, C. (2013) : Enseigner le lexique, Namur, Presses universitaires de Namur.

GOLDER, C. (1993) : « Savez-vous argumenter à la mode... à la mode des petits ? », Enfance, 46 (4), p. 359-376.

- (1996) : «La production de discours argumentatifs : revue de questions », Revue française de pédagogie, vol. 116, p. 119-134.

GUIBERT, R. (1998) : «Écriture du mémoire : exercice d'apprentissage de la complexité et de construction identitaire ", in : F. Cros, Le mémoire professionnel en formation des enseignants, Paris, Éd. L'Harmattan, p. 101-128.

GUIGUE, M. \& CRINON, J. (2003) : «Les modalités de présence des élèves dans des mémoires professionnels ", in : J. Crinon (dir.), Le mémoire professionnel des enseignants. Observatoire des pratiques et levier pour la formation, Paris, Éd. L'Harmattan, p. 113-132.

HOFSTETTER, R. \& SCHNEUWLY, B. (2009) : Savoirs en (trans)formation. Au cœur des professions de l'enseignement et de la formation, Bruxelles, De Boeck.

JAFFRÉ, J.-P. (1992) : Didactiques de l'orthographe, Paris, Hachette.

JAUBERT, M. \& REBIÈRE, M. (2010) : «Communautés discursives disciplinaires scolaires et formats d'interactions ", communication présentée au colloque international Spécificités et diversité des interactions didactiques : disciplines, finalités, contextes, 24-26 juin 2010, Université de Lyon/ICAR/ CNRS/INRP.

JOLIBERT, J. (2007) : Former des enfants producteurs de textes, Paris, Hachette.

LAFONT-TERRANOVA, J. (dir.) (2009) : Se construire, à l'école, comme sujet-écrivant : l'apport des ateliers d'écriture, Diptyque, 15.

LAHIRE, B. (1993) : Culture écrite et inégalités scolaires, Lyon, Presses universitaires de Lyon. - (2008) : La Raison scolaire. École et pratiques d'écriture, entre savoir et pouvoir, Rennes, Presses universitaires de Rennes. 
LEBRUN, M. (1996) : «Un outil d'appropriation du texte littéraire : le journal dialogué », in : , J.L. Dufays et al. (dirs), Pour une lecture littéraire, Bruxelles, De Boeck.

- (2004) : « Un nouveau contenu d'enseignement, la littérature à l'école primaire française : quelques interrogations ", actes du $9^{e}$ colloque de l'AIRDF, Québec.

Le Français aujourd'hui (1998) : 123 (1), « Argumenter : enjeux et pratiques ».

- (2003) : 140 (1), « Gestes et enjeux de la correction ».

- (2004) : 144 (1), « Réécritures ».

- (2004) : 145 (2), « Le littéraire et le social : visées critiques et place de l'affect ».

- (2004) : 147 (4), « Le biographique ».

- (2006) : 153 (2), « Enseigner l'écriture littéraire ».

- (2007) : 157 (2), « Sujet lecteur, sujet scripteur, quels enjeux pour la didactique? ».

- (2010) : 170 (3), « Graphies : signes, gestes, supports ».

- (2011) : 174 (3), « Penser à l'écrit ».

- (2012) : 178 (3), «L'enseignement des lettres et le numérique ».

- (2013) : 181 (2), « Écrits d'élèves, contraintes de la langue ».

LEPORCQ C. et al. (2013) : « Pratiques et représentations juvéniles de l'écriture à l'ère d'Internet », Cahiers de recherche du GIRSEF, 94.

MANESSE, D. \& COGIS, D. (2007) : Orthographe : à qui la faute ?, Paris, ESF.

MARTY, N. (2005) : Informatique et nouvelles pratiques d'écriture, Paris, Nathan.

NoËL, B. (1997) : La métacognition, Bruxelles, De Boeck.

NONNON, É. (1995) : « Les interactions lecture-écriture dans l'exposé d'une écriture professionnelle : le mémoire des professeurs débutants », Pratiques, 86, p. 93-122.

PENLOUP, M.-C. (1999) : L'écriture extrascolaire des collégiens, Paris, ESF.

PENLOUP M.-C., LIÉNARD, F. (2011) : « Le rapport à l'écriture, un outil pour penser la place de l'écriture électronique dans l'enseignement-apprentissage du français », Forumlecture.ch, 2 (en ligne : www.forumlecture.ch).

PIMET, O. \& BONIFACE, C. (1999) : Ateliers d'écriture, mode d'emploi, Paris, ESF.

PIOLAT, A. (dir.) (2004) : Écriture. Approches en sciences cognitives, Aix-en-Provence, Presses de l'université de Provence.

Pratiques, (1979) : 25, « Orthographe ».

- (1980) : 26, « Écrire en classe ».

- (1980) : 28, « Argumenter ».

- (1981) : 29, « La rédaction ».

- (1985) : 48, « Les écrits fictionnels».

- (1986) : 49, « Les activités rédactionnelles ».

- (1989) : 61, « Ateliers d'écriture ».

- (1990) : 68, « La dissertation ».

- (1992) : 73, «L'argumentation écrite».

- (1992) : 75, « Apprendre à rédiger ».

- (1994) : 83, «Écrire des récits».

- (1994) : 84, « Argumentation et langue ».

- (1995) : 86, « Lecture/écriture».

- (1997) : 96, « Enseigner l'argumentation».

- (2002) : 105-106, « La réécriture ».

- (2002) : 113-114, « Images du scripteur et rapports à l'écriture ». 
- (2002) : 115-116, «L'écriture et son apprentissage ».

- (2005) : 127-128, « L'écriture d'invention ».

- (2006) : 131-132, «La littératie. Autour de Jack Goody ».

- (2007) : 133-134, « Récits et disciplines scolaires».

- (2009) : 143-144, « Les écrits de savoir ».

- (2012) : 153-154, «Littéracies universitaires : nouvelles perspectives ».

- (2012) : 155-156, « Lexique et écriture ».

Recherches (2007) : 45, «Écritures de soi ».

- (2011) : 55, « Brouillons».

- (1994) : 10, « Écrire, réécrire ».

- (1995) : 11, « Écriture et traitement de texte ».

- (1996) : 13, « Lecture et écriture littéraire à l'école ».

- (2001) : 23, «Les pratiques extra-scolaires de lecture et d'écriture des élèves ».

- (2002) : 26-27, « L'écriture et son apprentissage à l'école élémentaire ».

- (2005) : 31, «L'évaluation en didactique du français : résurgence d'une problématique ».

- (2006) : 33, « La fiction et son écriture ».

- (2006) : 34, « L'écriture de soi ».

- (2008) : 38, «Dimensions socioculturelles de l'enseignement du français à l'école primaire ».

- (2009) : 40, « Écrire avec, sur, de la littérature ».

REUTER, Y. (2000) : Enseigner et apprendre à écrire, Paris, ESF.

SCHEEPERS, C. (2008) : « Former des enfants réflexifs », Repères, 38, p. 99-120.

- (2009) : Le travail de fin d'études, un discours en quête d'auteur. Pratiques langagières réflexives en formation initiale d'enseignants, thèse inédite, université de Liège/Université Paris 8 VincennesSaint-Denis.

- (2013) : L'argumentation écrite, Bruxelles, De Boeck.

- (2014) : «Les préparations de cours : apprendre à écrire, écrire pour faire apprendre, » Le français aujourd'hui, 184, p. 17-28.

Sciences humaines, (2000) : 109, «Les logiques de l'écriture ».

- (2013) : 253, «Écrire, du roman au SMS».

Sciences humaines. Grands dossiers, (2008) : 11, « Entre image et écriture ».

SCHNEEBERGER, P. \& VÉRIN, A. (2009) : Développer des pratiques d'oral et d'écrit en sciences, Paris, INRP.

SCHNEUWLY, B. (1988) : Le langage écrit chez l'enfant. La production de textes informatifs et argumentatifs , Lausanne, Delachaux et Niestlé.

SCHNEUWLY, B. \& DOLZ, J. (2006) : Des objets enseignés en classe de français. Le travail de l'enseignant sur la rédaction de textes argumentatifs et sur la subordonnée relative, Rennes, Presses universitaires de Rennes.

SÉGUY, A. (1994) : «Écrire et réécrire en classe, pour quoi faire ? : finalisation des écrits et critères de réécriture », Repères, 10, p. 13-32

SIMARD, C. (2012) : Didactique du français langue première, Bruxelles, De Boeck.

TAUVERON, C. \& SÈVE, P. (2005) : Vers une écriture littéraire. Ou comment construire une posture d'auteur à l'école de la GS au CM, Paris, Hatier.

TERWAGNE, S. et al. (2001) : Les cercles de lecture, Bruxelles, De Boeck.

THYRION, F. (2006) : La dissertation. Du lieu commun au texte de réflexion personnelle, Bruxelles, De Boeck. 
TENORTH, H.-E. (2009) : « Différence de statut : différence de standards pédagogiques », in :

R. Hofstetter \& B. Schneuwly (dirs) (2009), Savoirs en (trans)formation. Au cœur des professions de l'enseignement et de la formation, Bruxelles, De Boeck, p. 83-108.

VANHULLE, S. (2002) : Des savoirs en jeu au savoir en «je », thèse inédite, université de Liège.

- (2009) : Des savoirs en jeu au savoir en je. Cheminements réflexifs et subjectivation des savoirs chez de jeunes enseignants en formation, Berne, P. Lang.

VIANIN, P. (1999) : L'aide stratégique aux élèves en difficulté scolaire, Bruxelles, De Boeck.

VYGOTSKI, L. (1997 [1934]) : Pensée et langage, trad. du russe, Paris, Éd. La Dispute.

\section{NOTES}

1. Un projet de mastérisation est à l'étude depuis plusieurs années, mais sans qu'il soit suivi d'effets à l'heure actuelle.

2. Que soient ici remerciés les directeurs et les bibliothécaires qui ont facilité l'accès aux TFE produits dans leur établissement. Je précise que je ne suis en rien liée aux TFE qui constituent mon corpus : je n'ai été amenée ni à les évaluer, ni à les accompagner. En outre, je n'ai pas dispensé de cours aux étudiants dont j'ai étudié les TFE. Si je suis bien formatrice d'enseignants, c'est dans deux autres établissements, avec des publics différents, de futurs instituteurs du primaire d'une part, des étudiants universitaires en langues et lettres d'autre part.

3. Les bibliographies comptent d'une à cinq pages, elles en font deux en moyenne. Les bibliographies sont dans la majorité des cas structurées selon le type de source mentionnée : ouvrages, dictionnaires, périodiques, sources internet, TFE...

\section{RÉSUMÉS}

Quels sont les modèles didactiques dominants dans les discours des formateurs d'enseignants? Peut-on mettre au jour les discours tenus par ces formateurs à propos de l'écriture ? Quelle place l'écriture tient-elle dans les plans de formation? Plus spécifiquement, quels savoirs d'ordre scientifique, curriculaire, décrétal et expérienciel sont mobilisés par les futurs agrégés à propos de l'écriture dans le travail de fin d'études (mémoire) qui clôture leur formation initiale en haute école? Ainsi, le travail de fin d'études (TFE) s'envisage dans cette perspective comme un observatoire et un moteur privilégiés des savoirs qu'est supposé convoquer et façonner l'apprenti-enseignant pour concevoir, expérimenter, accompagner, réguler et évaluer un dispositif d'écriture destiné aux élèves du début du secondaire. L'analyse montre notamment que les travaux produits par les didacticiens de l'écriture sont peu convoqués par les étudiants dans le cadre de leur recherche.

What are the dominant didactic models in the speeches of the trainers of teachers? Can we bring to light the speeches held by these trainers about the writing? What places the writing does it hold in training plans? More specifically, what knowledge are mobilized by the future qualified teachers about the writing in the thesis which encloses their initial training? So, the thesis envisages in this perspective as an observatory and an engine favored by the knowledges which 
is supposed to summon and to shape the apprentice-teacher to conceive, experiment, accompany, to regulate and to estimate a device of writing intended for the pupils of the beginning of the high school. The analysis shows in particular that the works produced by the didacticiens of the writing are little summoned by the students within the framework of their research.

INDEX

Mots-clés : formation d'enseignants, mémoires, savoirs

Keywords : teacher training, thesis, knowledges

\section{AUTEUR}

CAROLINE SCHEEPERS

Haute École Lucia de Brouckère 